\title{
P-CLEAN RINGS
}

\author{
WEIXING CHEN
}

Received 20 March 2006; Accepted 21 March 2006

In this paper we unify the structures of various clean rings by introducing the notion of $P$-clean rings. Some properties of $P$-clean rings are investigated, which generalize the known results on clean rings, semiclean rings, $n$-clean rings, and so forth. By the way, we answer a question of Xiao and Tong on $n$-clean rings in the negative.

Copyright (c) 2006 Hindawi Publishing Corporation. All rights reserved.

\section{Introduction}

Throughout this paper $R$ denotes an associative ring with identity and all modules are unitary. We use the symbol $U(R)$ to denote the group of units of $R$ and $\operatorname{Id}(R)$ the set of idempotents of $R, U_{n}(R)$ the set of elements which are the sum of $n$ units of $R, U_{\Sigma}(R)$ the set of elements each of which is the sum of finitely many units in $R, R E(R)(U R E(R))$ the set of regular (unit regular) elements of $R$, and $\operatorname{Peri}(R)$ the set of periodic elements of $R$. The Jacobson radical and the prime radical of $R$ are denoted by $J(R)$ and $\mathrm{Nil}_{*}(R)$, respectively.

Following Han and Nicholson [4], an element $x$ of a ring $R$ is called clean if $x=e+u$ where $e \in \operatorname{Id}(R)$ and $u \in U(R)$. A ring $R$ is clean if every element of $R$ is clean. This notion was first introduced by Nicholson [5] as early as 1977 in his study of lifting idempotents and exchange rings. Since then, a great deal is known about clean rings and their generalizations (cf. [1-9]).

According to Ye [9], a ring $R$ is called semiclean if every element of $R$ has the form $x=f+u$, where $u \in U(R)$ and $f$ is periodic, that is, $f^{p}=f^{q}$ for two different positive integers $p$ and $q$. In [8], an element $x$ of a ring $R$ is called $n$-clean if $x=e+u_{1}+\cdots+u_{n}$ where $e \in \operatorname{Id}(R), u_{i} \in U(R)$, and $n$ is a positive integer. The ring $R$ is called $n$-clean if every element of $R$ is $n$-clean for some fixed positive integer $n$. While $R$ is called $\Sigma$-clean, if the $n$ is a positive integer depending on $x$. Also Zhang and Tong in [10] defined $R$ to be $G$-clean, if each $x \in R$ has the form $x=a+u$ where $a$ is unit regular and $u \in U(R)$.

Motivated by the results of Han and Nicholson [4] on clean rings, Ye [9] on semiclean rings, Xiao and Tong [8] on $n$-clean rings and $\Sigma$-clean rings, and Zhang and Tong [10] on $G$-clean rings, in this paper we unify the structures of various clean rings by introducing 
the notion of $P$-clean rings and the common properties of those rings. By the way, we answer a question of Xiao and Tong [8] in the negative and extend some known results of $[8,9]$.

\section{2. $P$-clean rings}

We start this section by the following definitions.

For two subsets $A$ and $B$ of a ring $R$, the sum of $A$ and $B$ is defined as follows: $A+$ $B=\{a+b \mid a \in A, b \in B\}$. The sum of more than two subsets of an $R$ can be defined inductively.

Let $P$ be a property which is meaningful for elements of a ring. For any $\operatorname{ring} R$, let $P(R)$ be the subset $\{a \in R \mid a$ has property $P\}$ of $R$.

Definition 2.1. Property $P$ will be called admissible if the following conditions are satisfied.

(1) For any ring homomorphism $\sigma: R \rightarrow S, \sigma(P(R)) \subseteq P(S)$.

(2) For any rings $R \subseteq S, P(R) \subseteq P(S)$.

(3) For any $e \in \operatorname{Id}(R), P(e R e)+P((1-e) R(1-e)) \subseteq P(R)$.

For convenience, an element of $P(R)$ is called a $P$-element in $R$. In this paper $P$ will always be an admissible property.

Proposition 2.2. (1) If $\sigma$ is a ring isomorphism from $R$ onto $S$, then $\sigma(P(R))=P(S)$.

(2) If $e_{1}, e_{2}, \ldots, e_{n}$ are orthogonal complete idempotents, that is, $e_{i} e_{j}=0$ whenever $i \neq j$ and $e_{i}^{2}=e_{i}$, and $e_{1}+e_{2}+\cdots+e_{n}=1$, then $P\left(e_{1} R e_{1}\right)+P\left(e_{2} R e_{2}\right)+\cdots+P\left(e_{n} R e_{n}\right) \subseteq$ $P(R)$.

Proof. (1) By Definition 2.1, $\sigma(P(R)) \subseteq P(S)$, hence $\sigma^{-1}(\sigma(P(R))) \subseteq \sigma^{-1}(P(S)) \subseteq P(R)$. It follows that $P(R) \subseteq \sigma^{-1}(P(S)) \subseteq P(R)$, which gives $\sigma(P(R)) \subseteq P(S) \subseteq \sigma(P(R))$ and so $\sigma(P(R))=P(S)$.

(2) We prove this by using induction on $n$. In fact, the case $n=2$ is condition (3) of Definition 2.1. Assume (2) holds for $n-1$. Let $e_{1}+e_{2}+\cdots+e_{n-1}=f$. Then multiplied by $e_{i}$ on the two sides of the above equation, we have $e_{i} f=f e_{i}=e_{i}$, which gives $e_{i}=f e_{i} f$ and so $e_{i} \in \operatorname{Id}(f R f)$. Note that $f R f$ is a ring with identity $f$. It yields that $P\left(e_{1} R e_{1}\right)+P\left(e_{2} R e_{2}\right)+\cdots+P\left(e_{n-1} R e_{n-1}\right) \subseteq P(f R f)$ by inductive assumption. On the other hand, $f+e_{n}=1$ implies $P(f R f)+P\left(e_{n} R e_{n}\right) \subseteq P(R)$ by Definition 2.1(3). Hence $P\left(e_{1} R e_{1}\right)+P\left(e_{2} R e_{2}\right)+\cdots+P\left(e_{n-1} R e_{n-1}\right)+P\left(e_{n} R e_{n}\right) \subseteq P(f R f)+P\left(e_{n} R e_{n}\right) \subseteq P(R)$.

Definition 2.3. A ring $R$ is called $P$-clean if every $x \in R$ has the form $x=p+u$, where $p \in P(R)$ and $u \in U(R)$.

Lemma 2.4. Let $R$ be a ring and $e \in \operatorname{Id}(R)$. Then the following hold.

(1) If $u \in U(e R e)$ and $v \in U((1-e) R(1-e))$, then $u+v \in U(R)$.

(2) If $e_{1} \in \operatorname{Id}(e R e)$ and $e_{2} \in \operatorname{Id}((1-e) R(1-e))$, then $e_{1}+e_{2} \in \operatorname{Id}(R)$.

(3) If $f \in \operatorname{Peri}(e R e)$ and $g \in \operatorname{Peri}((1-e) R(1-e))$, then $f+g \in \operatorname{Peri}(R)$.

(4) If $x \in R E(e R e)$ and $y \in R E((1-e) R(1-e))$, then $x+y \in R E(R)$.

(5) If $x \in U R E(e R e)$ and $y \in U R E((1-e) R(1-e))$, then $x+y \in U R E(R)$.

(6) If $x \in U_{n}(e R e)$ and $y \in U_{n}((1-e) R(1-e))$, then $x+y \in U_{n}(R)$. 
(7) If $x \in \operatorname{Id}(e R e)+U_{n}(e R e)$ and $y \in \operatorname{Id}((1-e) R(1-e))+U_{n}((1-e) R(1-e))$, then $x+y \in \operatorname{Id}(R)+U_{n}(R)$.

(8) If $x \in \operatorname{Id}(e R e)+U_{\Sigma}(e R e)$ and $y \in \operatorname{Id}((1-e) R(1-e))+U_{\Sigma}((1-e) R(1-e))$, then $x+y \in \operatorname{Id}(R)+U_{\Sigma}(R)$.

Proof. We only prove (3) and (8), the others are very similar.

(3) Let $f \in \operatorname{Peri}(e R e)$ and $g \in \operatorname{Peri}((1-e) R(1-e))$. Then there exist positive integers $m>n$ and $p>q$ such that $f^{m}=f^{n}$ and $g^{p}=g^{q}$. By Ye [9, Lemma 5.2], $f^{n(m-n)}$ and $g^{q(p-q)}$ are both idempotents. Set $t=2 n(m-n) q(p-q)$. Then $f^{2 t}=f^{t}$ and $g^{2 t}=g^{t}$. Since $f g=g f=0,(f+g)^{2 t}=f^{2 t}+g^{2 t}=f^{t}+g^{t}=(f+g)^{t}$. Hence $f+g \subseteq \operatorname{Peri}(R)$.

(8) Assume $x \in \operatorname{Id}(e R e)+U_{\Sigma}(e R e)$ and $y \in \operatorname{Id}((1-e) R(1-e))+U_{\Sigma}((1-e) R(1-e))$. Then $x=f+u_{1}+\cdots+u_{n}$ and $y=g+v_{1}+\cdots+v_{m}$ where $f \in \operatorname{Id}(e R e), g \in \operatorname{Id}((1-$ $e) R(1-e)), u_{i} \in U(e R e)$, and $v_{j} \in U((1-e) R(1-e))$. It is easy to show that an $n$-clean element is $m$-clean whenever $n \leq m$, since for any $e \in \operatorname{Id}(R), e=(1-e)+(2 e-1)$ where $1-e \in \operatorname{Id}(R)$ and $(2 e-1)^{2}=1$. So without loss of generality, we can assume $n=m$. Using (1), $f+g \in \operatorname{Id}(R)$. And from (6), $u_{i}+v_{i} \in U(R)$, hence $x+y=(f+g)+\left(u_{1}+v_{1}\right)$ $+\cdots+\left(u_{n}+v_{n}\right) \in \operatorname{Id}(R)+U_{\Sigma}(R)$.

Using Lemma 2.4, it is easy to check that for any $\operatorname{ring} R, 0, R, \operatorname{Id}(R), \operatorname{Peri}(R), U(R)$, $R E(R), U R E(R), U_{n}(R), \operatorname{Id}(R)+U_{n-1}(R)$ for $n \geq 2, \operatorname{Id}(R)+U_{\Sigma}(R)$ are all subsets of $R$ defined by a suitable admissible property $P$.

From the above arguments, the following proposition is immediate.

Proposition 2.5. Let $R$ be a ring. Then the following conclusions hold.

(1) $\operatorname{Id}(R)$-clean rings are precisely clean rings.

(2) Pri $(R)$-clean rings are precisely semiclean rings.

(3) $U(R)$-clean rings are precisely $(S, 2)$-rings.

(4) $U r e(R)$-clean rings are precisely $G$-clean rings.

(5) $\operatorname{Id}(R)+U_{n-1}(R)$-clean rings are precisely $n$-clean rings when $n \geq 2$.

(6) $\operatorname{Id}(R)+U_{\Sigma}(R)$-clean rings are precisely $\Sigma$-clean rings.

Note that here an $(S, 2)$-ring is a ring in which every element can be expressed as a sum of two units of $R$. While in some literature it referred to a ring in which every element can be written as a sum of no more than two units.

Proposition 2.6. Any homomorphic image of a P-clean ring is P-clean.

Proof. Let $R$ be a $P$-clean ring and let $f: R \rightarrow S$ be a ring surjective homomorphism. Then for any $y \in S$, there exists $x \in R$ such that $f(x)=y$. Since $R$ is $P$-clean, $x=p+u$ with $p \in P(R)$ and $u \in U(R)$. Hence $f(x)=f(p)+f(u)$. Obviously $f(u) \in U(S)$ and $f(p) \in f(P) \subseteq P(S)$ by Definition 2.1, the proof is complete.

Proposition 2.7. A finite direct product $R=\prod_{i=1}^{n} R_{i}$ of rings $R_{i}$ is $P$-clean if and only if each $R_{i}$ is $P$-clean.

Proof. If $R$ is $P$-clean, then each $R_{i}$ is $P$-clean by Proposition 2.6. Conversely, assume each $R_{i}$ is $P$-clean, and $x=\left(x_{i}\right) \in R$. Then $x_{i}=p_{i}+u_{i}$ with $p_{i} \in P\left(R_{i}\right)$ and $u_{i} \in U\left(R_{i}\right)$ 
for each $i$. By Proposition 2.2, we can identify $R_{i}$ with $\left(\ldots, 0, R_{i}, 0, \ldots\right)$ canonically. Let $e_{i}=$ $(\ldots, 0,1,0, \ldots)$. Then $\left(p_{i}\right)=\left(p_{1}, 0, \ldots, 0\right)+\left(0, p_{2}, \ldots, 0\right)+\cdots+\left(0,0, \ldots, p_{n}\right) \in P\left(e_{1} R e_{1}\right)+$ $P\left(e_{2} R e_{2}\right)+\cdots+P\left(e_{n} P e_{n}\right) \subseteq P(R)$. Now $x=\left(x_{i}\right)=\left(p_{i}+u_{i}\right)=\left(p_{i}\right)+\left(u_{i}\right)$ with $\left(p_{i}\right) \in P(R)$ and $\left(u_{i}\right) \in U(R)$, so we are done.

It should be noted that Proposition 2.7 is not true for an infinite direct product of rings $R_{i}$. For example, the ring $\mathbb{Z}$ of integers is a $\sum$-clean ring, but $R=\prod_{i=1}^{\infty} \mathbb{Z}$ is not $\Sigma$ clean since $(1,2, \ldots, n, \ldots)$ is obviously not $\sum$-clean.

Proposition 2.8. The ring $R$ is $P$-clean if and only if the ring $R[[x]]$ of formal power series over $R$ is $P$-clean.

Proof. If $R[[x]]$ is $P$-clean, then $R$ is $P$-clean by Proposition 2.6. Now if $R$ is $P$-clean, then for any $f(x) \in R[[x]], f(x)=a_{0}+a_{1} x+\cdots+a_{n} x^{n}+\cdots$. By assumption, $a_{0}=p+u$ with $p \in P(R)$ and $u \in U(R)$. Hence $f(x)=p+u+a_{1} x+\cdots+a_{n} x^{n}+\cdots$ with $p \in P(R) \subseteq$ $P(R[[x]])$ and $u+a_{1} x+\cdots+a_{n} x^{n}+\cdots \in U(R[[x]])$, as desired.

The following corollary extends [8, Proposition 2.5] which states that for a commutative ring $R, R$ is $n$-clean if and only if $R[[x]]$ is $n$-clean.

Corollary 2.9. $R$ is $n$-clean ( $\sum$-clean) if and only if $R[[x]]$ is $n$-clean ( $\Sigma$-clean).

It has been proved by Han and Nicholson in [4] that if $e$ is an idempotent in a ring $R$ such that $e$ Re and $(1-e) R(1-e)$ are both clean rings, then $R$ is clean. Hence the ring of $n \times n$ matrices over $R$ is clean. Similar results hold for semiclean rings, $n$-clean rings, and $\Sigma$-clean rings. We now extend these results to $P$-clean rings.

Lemma 2.10. Let $e \in \operatorname{Id}(R)$ be such that $e$ Re and $(1-e) R(1-e)$ are both $P$-clean rings. Then $R$ is a $P$-clean ring.

Proof. For convenience, write $\bar{r}=1-r$ for each $r \in R$. We use the Pierce decomposition of the ring $R$ :

$$
R=e R e+e R \bar{e}+\bar{e} R e+\bar{e} R \bar{e} .
$$

Let $x=a+b+c+d$ where $a \in e R e, b \in e R \bar{e}, c \in \bar{e} R e$, and $d \in \bar{e} R \bar{e}$. By hypothesis, write $a=p+u$ where $p \in P(e R e)$ and $u \in U(e R e)$ with inverse $u_{1}$. Then $d-c u_{1} b \in \bar{e} R \bar{e}$, so write $d-c u_{1} b=q+v$ where $q \in P(\bar{e} R \bar{e})$ and $v \in U(\bar{e} R \bar{e})$ with inverse $v_{1}$. Hence $x=$ $(p+q)+u+b+c+v+c u_{1} b$ and it suffices to show that $u+b+c+v+c u_{1} b$ is a unit in $R$. To this end compute

$$
\begin{aligned}
&\left(u+b+c+v+c u_{1} b\right)\left(u_{1}+u_{1} b v_{1} c u_{1}-u_{1} b v_{1}-v_{1} c u_{1}+v_{1}\right) \\
&=\left(e+b v_{1} c u_{1}-b v_{1}\right)+\left(-b v_{1} c u_{1}+b v_{1}\right)+\left(c u_{1}+c u_{1} b v_{1} c u_{1}-c u_{1} b v_{1}\right) \\
&+\left(-c u_{1}+1-e\right)+\left(-c u_{1} b v_{1} c u_{1}+c u_{1} b v_{1}\right)=1 .
\end{aligned}
$$

Similarly, $\left(u_{1}+u_{1} b v_{1} c u_{1}-u_{1} b v_{1}-v_{1} c u_{1}+v_{1}\right)\left(u+b+c+v+c u_{1} b\right)=1$.

Note that $p+q \in P(e R e)+P(\bar{e} R \bar{e}) \subseteq P(R)$ by Definition 2.1, the proof is complete.

Using Lemma 2.10, an inductive argument gives immediately. 
Theorem 2.11. If $1=e_{1}+e_{2}+\cdots+e_{n}$ in a ring $R$ where $e_{i}$ are orthogonal idempotents and each $e_{i} R e_{i}$ is $P$-clean, then $R$ is $P$-clean.

The following two results are direct consequences of Theorem 2.11

Corollary 2.12. If $R$ is a $P$-clean ring, so also is the matrix ring $M_{n}(R)$.

Corollary 2.13. If $M=M_{1} \oplus M_{2} \oplus \cdots \oplus M_{n}$ are modules and end $\left(M_{i}\right)$ is $P$-clean for each $i$, then end $(M)$ is P-clean.

Since any homomorphic image of a $P$-clean ring is again $P$-clean, with Theorem 2.11, this gives the following.

Corollary 2.14. If $A$ and $B$ are rings and $V={ }_{A} V_{B}$ is a bimodule, the split-null extension $R$ is $P$-clean if and only if both $A$ and $B$ are $P$-clean, where

$$
R=\left(\begin{array}{ll}
A & V \\
0 & B
\end{array}\right) .
$$

In particular, induction shows that for each $n \geq 1$, a ring $R$ is $P$-clean if and only if the ring of all $n \times n$ upper triangular matrices over $R$ is $P$-clean.

Let $R$ be a ring and let $I$ be an ideal of $R$. We say $P$-elements in $R / I$ lift modulo $I$, if for any $p \in P(R / I)$ there exists $a \in P(R)$ such that $\pi(a)=p$ where $\pi$ is the canonical ring homomorphism from $R$ onto $R / I$.

We close this section with the following proposition whose proof is very easy.

Proposition 2.15. Let $R$ be a ring and let I be an ideal contained in $J(R)$. If $R / I$ is a $P$-clean ring and $P$-elements lift modulo $I$, then $R$ is a P-clean ring.

\section{Some remarks}

It is known by [4, Proposition 6] that a ring $R$ is clean if and only if $R / I$ is clean for any ideal $I \subseteq J(R)$ and idempotents lift modulo $I$. Xiao and Tong [8] naturally claimed that they do not know whether for any $n$-clean ring $R$, idempotents of $R / I$ lift modulo $I$ where $I$ is any ideal of $R$ contained in $J(R)$. The following counterexample shows that the answer is negative.

Example 3.1. There is a 4-clean ring $R$ in which idempotents of $R / J(R)$ cannot be lifted to $R$.

Proof. Let $R$ be the subring of rational numbers $Q$ given by $R=\{m / n \in Q \mid(m, n)=$ $(n, 6)=1\}$. Then $R$ has only two maximal ideals: $2 R$ and $3 R$, so $J(R)=6 R$. Denote the ring of integers modulo $n$ by $\mathbb{Z}_{n}$, then $R / J(R) \cong \mathbb{Z}_{2} \times \mathbb{Z}_{3}$, which has four idempotents. But $R$ has only two idempotents. This shows that idempotents of $R / J(R)$ cannot be lifted to $R$ modulo $J(R)$. But it can be shown that $R$ is a 4 -clean ring.

Clearly, $x=m / n \in U(R)$ if and only if $(m, 6)=(n, 6)=1$. Now for any $x \in R, x$ has the form $x=3^{p} 2^{q} m / n$ where $(m, 6)=(n, 6)=1$. If $p, q \geq 1$, then $3^{p} 2^{q} m=\left(3^{p} 2^{q}-1+1\right) m=$ $\left(3^{p} 2^{q}-1\right) m+m \in U_{2}(R)$, so $x \in U_{2}(R)$. If $x=3^{p} m / n$ with $p \geq 1$ and $(m, 6)=(n, 6)=1$, then $3^{p} m=\left(3^{p}-2+2\right) m=\left(3^{p}-2\right) m+m+m$, which implies $x \in U_{3}(R)$. Similarly, in 
the case of $x=2^{q} m / n$ where $(m, 6)=(n, 6)=1$ and $q \geq 1$, then $2^{q}=2^{q}-3+3=2^{q}-3+$ $1+1+1$. It follows that $x \in U_{4}(R)$. Since any $n$-clean element must be $m$-clean for any $n \leq m$ (cf. the proof of Lemma 2.4(8)), $R$ is a 4-clean ring from the above arguments. This ring $R$ is clearly a $k$-clean for any $k \geq 4$ as the proof shows.

The following two results are obtained by Xiao and Tong [8] for commutative rings, now we extend them to 2-primal rings (rings whose prime radical coincides with the set of nilpotent elements).

Proposition 3.2. For any 2-primal ring $R$, the polynomial ring $R[x]$ is not $\Sigma$-clean.

Proof. Assume the contrary, then $x=e(x)+u_{1}(x)+u_{2}(x)+\cdots+u_{n}(x)$ where $e(x) \in$ $\operatorname{Id}(R[x]), u_{i}(x) \in U(R[x])$ for each $i$, and $n$ is a positive integer. Let

$$
\begin{gathered}
e(x)=e_{0}+a_{1} x+\cdots+a_{m} x^{m}, \\
u_{1}(x)=u_{10}+u_{11} x+\cdots+u_{1 m} x^{m}, \\
\vdots \\
u_{n}(x)=u_{n 0}+u_{n 1} x+\cdots+u_{n m} x^{m} .
\end{gathered}
$$

Since $R$ is 2-primal, a polynomial over $R$ is invertible if and only if its constant term is in $U(R)$ and the other coefficients are in $\operatorname{Nil}_{*}(R)$ by [3, Theorem 2.4], so $u_{i j} \in \mathrm{Nil}_{*}(R)$ for each $j \geq 1$. Hence $x=e(x)+u_{1}(x)+\cdots+u_{n}(x)$ gives $a_{1}+u_{11}+\cdots+u_{n 1}=1$, so $a_{1}$ is a unit in $R$, and $a_{2}+u_{12}+\cdots+u_{n 2}=0$ implies $a_{2} \in \operatorname{Nil}_{*}(R)$. On the other hand, $e(x)^{2}=$ $e(x)$ implies $e_{0}^{2}=e_{0}$, and $e(x)^{2}=e_{0}+\left(e_{0} a_{1}+a_{1} e_{0}\right) x+\left(e_{0} a_{2}+a_{1}^{2}+a_{2} e_{0}\right) x^{2}+\cdots+a_{m}^{2} x^{2 m}$. So $a_{2}=e_{0} a_{2}+a_{1}^{2}+a_{2} e_{0}$ by comparing the coefficient of $x^{2}$ in $e(x)^{2}=e(x)$. Note that the sum of a unit and a nilpotent element must be a unit and $e_{0} a_{2}+a_{2} e_{0} \in \mathrm{Nil} *(R)$. It follows that $a_{2} \in U(R)$. This is a contradiction, and the proof is complete.

From Proposition 3.2, the following corollary is immediate.

Corollary 3.3. For any 2-primal ring $R$, the polynomial ring $R[x]$ is not $n$-clean.

We conclude this paper with the following proposition.

Proposition 3.4. For any 2-primal ring $R$, the polynomial ring $R[x]$ is not semiclean.

Proof. Assume the contrary, then $x=p(x)+u(x)$ where $p(x)$ is a periodic element and $u(x)$ is a unit. Let $p(x)=p_{0}+p_{1} x+\cdots+p_{n} x^{n}$ and $u(x)=u_{0}+u_{1} x+\cdots+u_{n} x^{n}$. Since $R$ is 2-primal, $u_{i} \in \mathrm{Nil}_{*}(R)$ for each $i \geq 1$ by [3, Theorem 2.4]. By comparing the coefficient of $x=p(x)+u(x)$, we have $p_{1}+u_{1}=1$, which implies $p_{1}$ is a unit in $R$, and $p_{i}+u_{i}=0$ gives $p_{i} \in \mathrm{Nil}_{*}(R)$ for each $i \geq 2$. Clearly we can assume that $p(x)^{s}=p(x)^{t}$ for positive integers $s>t \geq 2$. Then a routine calculation shows that the coefficient of $x^{s}$ in $p(x)^{s}$ is

$$
\sum_{i_{1}+i_{2}+\cdots+i_{s}=s} p_{i_{1}} p_{i_{2}} \ldots p_{i_{s}}=p_{1}^{s}+a \quad \text { for some } a \in \operatorname{Nil}_{*}(R) .
$$


While the coefficient of $x^{s}$ in $p(x)^{t}$ is

$$
\sum_{j_{1}+j_{2}+\cdots+j_{t}=s} p_{j_{1}} p_{j_{2}} \ldots p_{j_{t}}=b \quad \text { for some } b \in \mathrm{Nil}_{*}(R) .
$$

Comparing the coefficients of $x^{s}$ on two sides of $p(x)^{s}=p(x)^{t}$, we have $p_{1} \in \mathrm{Nil}_{*}(R)$, which is a contradiction.

The above result is obtained by Ye [9] only for a commutative ring.

\section{Acknowledgments}

This research was partially supported by the Doctorate Foundation of China Education Ministry (Grant no. 20020284009). The author wishes to thank his advisor Professor Wenting Tong for his helpful suggestions. Also he wishes to thank the referees for valuable comments.

\section{References}

[1] D. D. Anderson and V. P. Camillo, Commutative rings whose elements are a sum of a unit and idempotent, Communications in Algebra 30 (2002), no. 7, 3327-3336.

[2] V. P. Camillo and H.-P. Yu, Exchange rings, units and idempotents, Communications in Algebra 22 (1994), no. 12, 4737-4749.

[3] W. Chen, A note on polynomial rings, to appear in Southeast Asian Bulletin of Mathematics.

[4] J. Han and W. K. Nicholson, Extensions of clean rings, Communications in Algebra 29 (2001), no. 6, 2589-2595.

[5] W. K. Nicholson, Lifting idempotents and exchange rings, Transactions of the American Mathematical Society 229 (1977), 269-278.

[6] Strongly clean rings and Fitting's lemma, Communications in Algebra 27 (1999), no. 8, 3583-3592.

[7] K. Samei, Clean elements in commutative reduced rings, Communications in Algebra 32 (2004), no. 9, 3479-3486.

[8] G. Xiao and W. Tong, $n$-clean rings and weakly unit stable range rings, Communications in Algebra 33 (2005), no. 5, 1501-1517.

[9] Y. Ye, Semiclean rings, Communications in Algebra 31 (2003), no. 11, 5609-5625.

[10] H. B. Zhang and W. Tong, Generalized clean rings, Journal of Nanjing University Mathematical Biquarterly 22 (2005), no. 2, 183-188.

Weixing Chen: Mathematics and Information Science School, Shandong Institute of Business and Technology, Yantai 264005, China

E-mail address: wxchen5888@163.com 


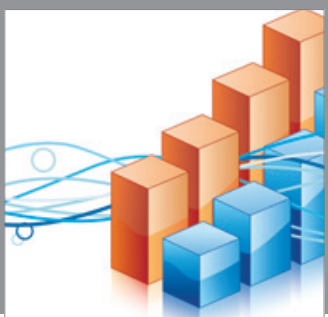

Advances in

Operations Research

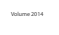

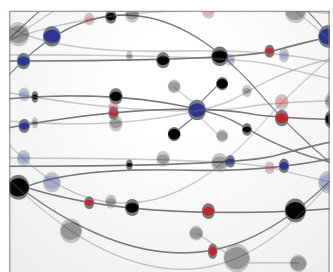

\section{The Scientific} World Journal
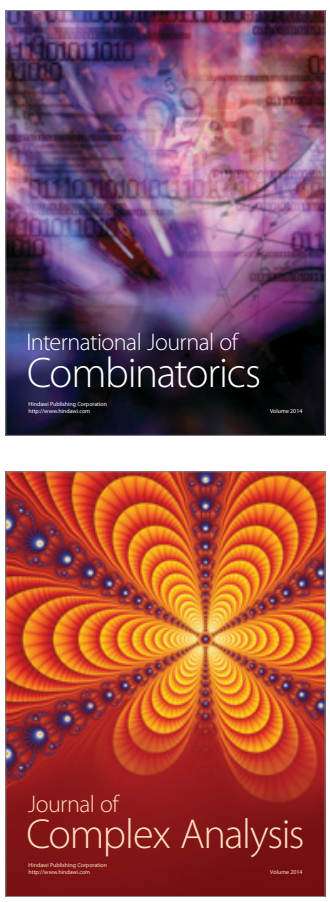

International Journal of

Mathematics and

Mathematical

Sciences
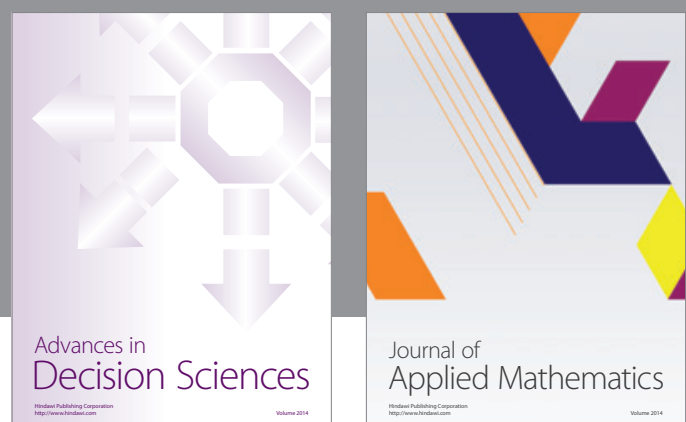

Journal of

Applied Mathematics
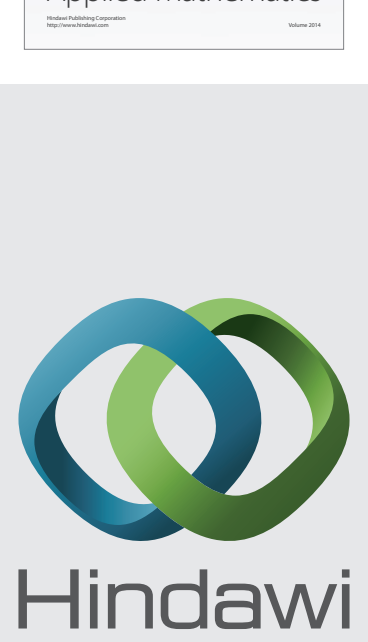

Submit your manuscripts at http://www.hindawi.com
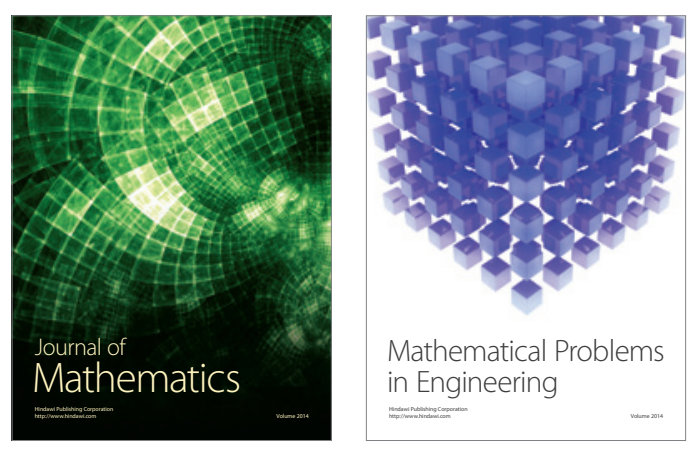

Mathematical Problems in Engineering
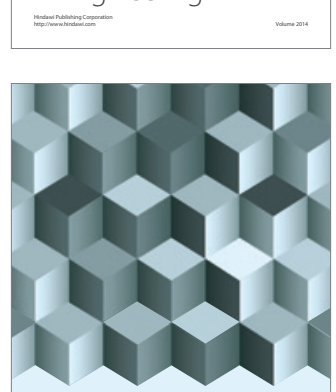

Journal of

Function Spaces
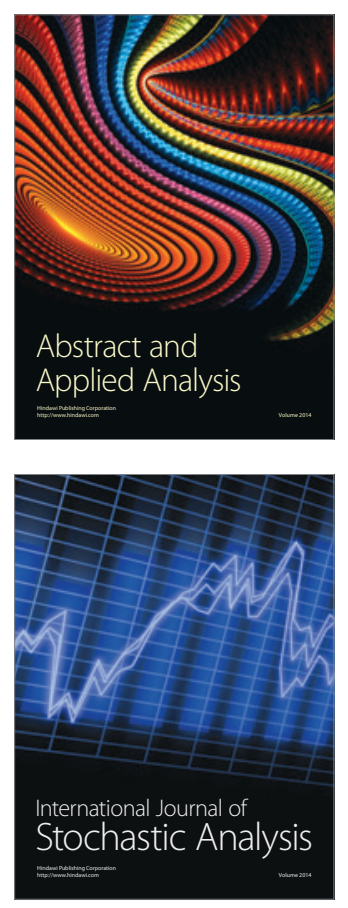

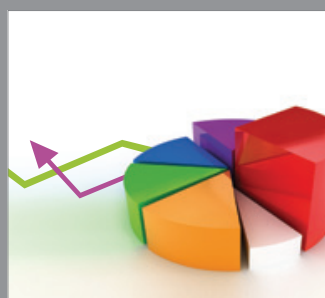

ournal of

Probability and Statistics

Promensencen
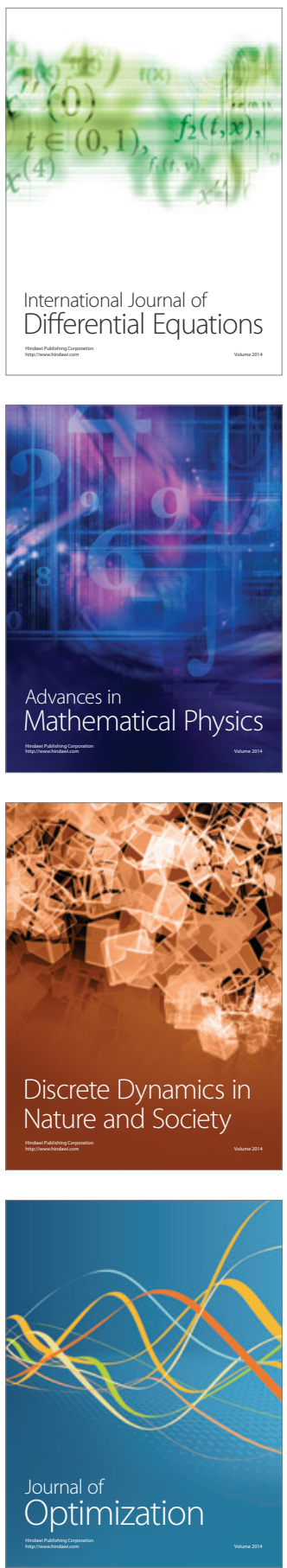